\title{
Perspective
}

PERSPECTIVE Actualité en histoire de l'art

$2 \mid 2015$

Les États-Unis

\section{Museum ad nauseam ? Des musées dans le labyrinthe postmoderne}

\section{Serge Guilbaut}

Traducteur : Isabelle Dubois

URL : http://journals.openedition.org/perspective/6146

DOI : $10.4000 /$ perspective.6146

ISSN : 2269-7721

\section{Éditeur}

Institut national d'histoire de l'art

Édition imprimée

Date de publication : 31 décembre 2015

Pagination : 13-26

ISBN : 978-2-917902-27-1

ISSN : $1777-7852$

\section{Référence électronique}

Serge Guilbaut, « Museum ad nauseam ? Des musées dans le labyrinthe postmoderne », Perspective [En ligne], 2 | 2015, mis en ligne le 30 juin 2017, consulté le 01 octobre 2020. URL : http://

journals.openedition.org/perspective/6146; DOI : https://doi.org/10.4000/perspective.6146 


\section{Museum ad nauseam ? Des musées dans le labyrinthe postmoderne}

En 1956, alors que la France commençait juste à profiter des avantages de la société de consommation, Charles Estienne, critique d'art reconnu et influent, qui défendait les formes les plus avancées de l'art moderne, décida de mettre fin définitivement à sa carrière dans le monde de l'art pour se consacrer à l'écriture de chansons populaires, en particulier pour son ami anarchiste Léo Ferré, et devint rapidement l'incarnation de la chanson à textes française.

Pour justifier un changement d'orientation aussi radical, Estienne, qui avait longtemps cru que la critique d'art était essentielle pour le développement d'une prise de conscience du public, expliquait que, face à la perte de sens progressive de la critique, souvent liée de trop près au marché de l'art et à la politique, il était alors d'un point de vue éthique préférable de prendre ses distances, tout en fredonnant une de ses propres ballades. Les espoirs et les aspirations qu'Estienne avait portés depuis la guerre s'étaient fracassés contre le mur ancestral du monde de l'art : celui de l'argent et de la célébrité. II avait découvert (ou redécouvert) que la signification de l'art était effectivement fluctuante et que, en tant que critique d'art, il n'avait que très peu de prise sur sa profession. Plus même, la conception en laquelle il avait toujours cru - à savoir que la critique était une sorte d'ange annonciateur de l'ère moderne - avait en réalité été transformée en l'image d'un petit boutiquier qui n'a pas grand-chose à vendre. Il avait compris qu'il ne pourrait pas échapper au destin peu enviable qui avait métamorphosé le critique d'art en publicitaire. Basculer comme il l'avait fait de la production de l'art pour l'élite à l'art populaire était sûrement problématique, mais au moins Estienne pensait pouvoir se soustraire, dans sa nouvelle situation, aux illusions que véhiculait encore le terme de « beaux-arts ». Il pourrait désormais participer pleinement à la vie quotidienne française sans avoir honte.

Charles Estienne, encore proche d'André Breton en 1955 et à la recherche d'un espace subversif à l'intérieur de la culture française, fut l'un des rares qui décida de se retirer de la « rat race » sans fin, comme elle était appelée alors. D'autres critiques d'art et d'autres musées, malgré les critiques grandissantes qui émanaient de groupes comme les lettristes ou les situationnistes, poursuivaient de bon train leurs activités liées à la promotion économique et politique. À la différence des critiques d'art qui avaient rapidement été transformés en simples recenseurs sans grande indépendance d'esprit (beaucoup de critiques à la fin des années 1950 étaient en fait employés par les galeries d'art : Michel Tapié, Clement Greenberg), les musées d'art moderne prospéraient, se multipliaient partout dans le monde occidental, devenant partie prenante du grand cirque quotidien ainsi que le décrivait Guy Debord, devenant des célébrités parmi tant d'autres.
Serge Guilbaut est professeur émérite d'histoire de l'art à I'University of British Columbia à Vancouver. Il a écrit sur l'art moderne et contemporain et, en particulier, sur les relations culturelles et politiques entre les États-Unis et la France.

Outre de nombreuses publications (dont How New York Stole the Idea of Modern Art: Abstract Expressionism, Freedom and the Cold War, Chicago, 1983 ; traduit en cinq langues) et des expositions dont il a été le commissaire (Théodore Géricault: The Alien Body, Tradition in Chaos, Vancouver, 1997 ; et Be-Bomb: The Transatlantic War of Images and All That Jazz, 1946-1956,

Barcelone, 2007), il a participé en tant qu'artiste à plusieurs expositions, dont une rétrospective à Vancouver en 2012 intitulée RetroPerspective (art et performances de 1965 à 2012). 


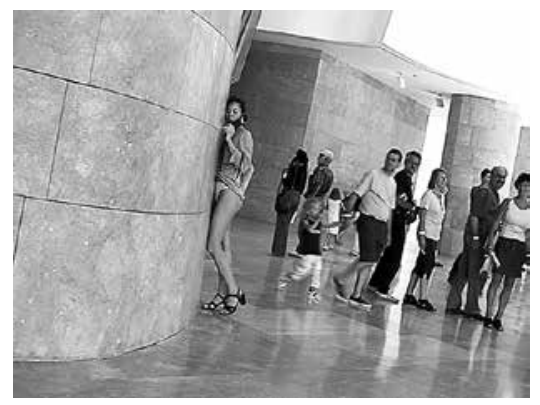

Je ne suis pas contre les musées, loin de là, puisque je collabore avec certains d'entre eux de temps à autre, pour articuler des démonstrations et des argumentations visuelles sur des moments d'histoire et de culture. Je ne suis pas contre le concept de musée en soi, et je ne suis certainement pas opposé à l'idée du musée comme lieu démocratique où les mouvements culturels sont présentés et discutés, où les notions de mémoire et d'histoire dialoguent afin d'éviter le piège qu'est le mythe d'une culture unique et transcendante. En revanche, je m'oppose au concept de musée comme source de profit, de prestige, et contre le musée comme espace de divertissement. Je suis contre le musée comme logo ou comme marque, contre le musée comme merveille architecturale, facilement reconnaissable dans le paysage urbain mais

1. Extrait de la video d'Andrea Fraser, Little Frank and his Carp, 2001. dépourvu d'idées : contre, en d'autres termes, le musée comme symbole vide de sens.

Évidemment nous savons depuis longtemps que les villes importantes ont toujours essayé de construire des musées reflétant leurs ambitions, mais dernièrement, depuis une vingtaine d'années, on assiste à une course effrénée entre les grandes villes du monde occidental pour se doter de lieux culturels affichant des architectures extravagantes pour attirer le touriste. L'architecture flamboyante d'architectes à la mode se veut immédiatement reconnaissable, même si le contenu en est pauvre et les collections quasi inexistantes, ainsi que Jean Clair l'a fait remarquer et l'a critiqué dans son livre Malaise dans les musées : "Malraux a inventé le musée imaginaire, le musée sans murs. Maintenant nous entrons dans l'ère des musées sans collections $»^{1}$. Les murs de ces nouveaux musées vides sont d'ailleurs magnifiques et ont tellement de succès que, à la manière d'Hollywood produisant des suites aux films à succès, des imitations de musées ont poussé dans de nombreuses villes à la recherche d'un label touristique. (Rappelez-vous la performance de l'artiste Andrea Fraser, caressant sensuellement les murs du musée Guggenheim à Bilbao [fig. 1].)

Mais même si mes propos résonnent comme ceux d'un vieux professeur ronchon, je ne pense pas être de ceux, comme Jean Clair, qui expriment de la nostalgie. Bien que j'approuve sa description du problème rencontré par le monde des musées d'aujourd'hui, je suis loin d'être d'accord avec sa solution, qui consiste à revenir en arrière vers une sorte $d^{\prime}$ espace élitiste, où seul I'art avec un grand $A$, choisi par des connaisseurs esthètes, serait exposé. Mais son diagnostic de la situation est, je pense, exact. La prolifération des visiteurs dans les musées modernes, qui aurait pu être une force, n'a néanmoins pas été suivie de progrès dans l'éducation artistique à l'école ni à l'université et certainement pas plus dans les musées eux-mêmes malgré la présence de services éducatifs, dont les actions sont trop souvent limitées au très jeune public. Nous avons donc désormais un public nouveau - très nombreux - confronté aux anciennes structures muséographiques encore destinées à un public de connaisseurs. Le résultat en est une rupture déplorable que Jean Clair souligne ainsi : "Moins nous comprenons les images plus nous nous précipitons pour les dévorer des yeux $»^{2}$. Le fonctionnement des musées publics se calque alors sur celui des entreprises privées, soucieuses de la gestion de leurs actifs ou, comme le disaient Jean-Pierre Jouyet et Maurice Lévy, auteurs du rapport sur "L'économie de l'immatériel ", "d'un management dynamique de leur capital immatériel $»^{3}$. Dans une économie globale, il est clair que le rôle d'un musée n'est plus d'entrer en dialogue avec le public, d'amorcer la mémoire individuelle ou nationale, de provoquer une prise de conscience historique nationale, mais plutôt de servir une image de marque (MoMA, Guggenheim, Louvre, Beaubourg, en France ou aux États-Unis).

Pendant des décennies, les musées ont fonctionné comme des espaces intimes, presque privatifs, pour des amateurs qui voulaient passer du temps à contempler des objets qu'ils connaissaient déjà. Les musées étaient comme des églises, 
les œuvres d'art des icônes adorées en silence. Toute l'organisation muséale constituait une consolidation du discours canonique établi par des conservateurs formalistes, entraînés à répéter sans fin la "messe ", à quelques exceptions près ${ }^{4}$. Cette organisation était protégée par un système de régulation solide et complexe, imposé par les conservateurs et les surveillants de musée. Ces derniers empêchaient les gens de parler ou de rire dans le sanctuaire, tandis que les conservateurs organisaient les expositions en reproduisant les valeurs traditionnelles transmises pendant leur formation. Bien que certains continuent à dire que la profession a changé, il s'avère que ces changements ne sont guère perceptibles dans les grandes expositions, dans leur majorité toujours aussi formalistes, biographiques, déconnectées de la réalité, de I'histoire et de la théorie. Pour se faire une idée de l'étendue du problème, il suffit de se rappeler que l'équivalent en français du terme anglais de curator est " conservateur", ce qui veut bien dire ce que ça veut dire ; les " conservateurs" sont bien conservateurs. L'alternative à cette appellation est " commissaire (d'exposition) ", ce qui ne suggère pas l'ouverture d'esprit et le sens de l'expérimentation. De même, quand on se souvient que curator en vieil anglais signifie une personne qui s'occupe des malades mentaux..., on a le sentiment que cette profession doit supporter une lourde charge symbolique!

Il est difficile de nos jours de continuer à défendre la vision persistante d'Alfred Barr, celui-là même qui a réussi à convaincre l'ensemble du monde occidental de l'importance d'alimenter l'idée fondamentale d'une progression moderniste des arts. Pendant des années, les musées ont accepté le schéma de Barr montrant que l'art moderne de haut niveau évoluait rapidement, innovait constamment et se perfectionnait lui-même en avançant énergiquement avec la rapidité d'une torpille. Si son graphique/ torpille de 1933 montrait le développement du mouvement artistique en partant 2a. Alfred Hamilton Barr Jr., couverture du catalogue de I'exposition Cubism and Abstract Art, New York, The Museum of Modern Art Library, MA143 ;

b. graphique de la collection idéale, New York, The Museum of Modern Art Library, New York, 9a.16.
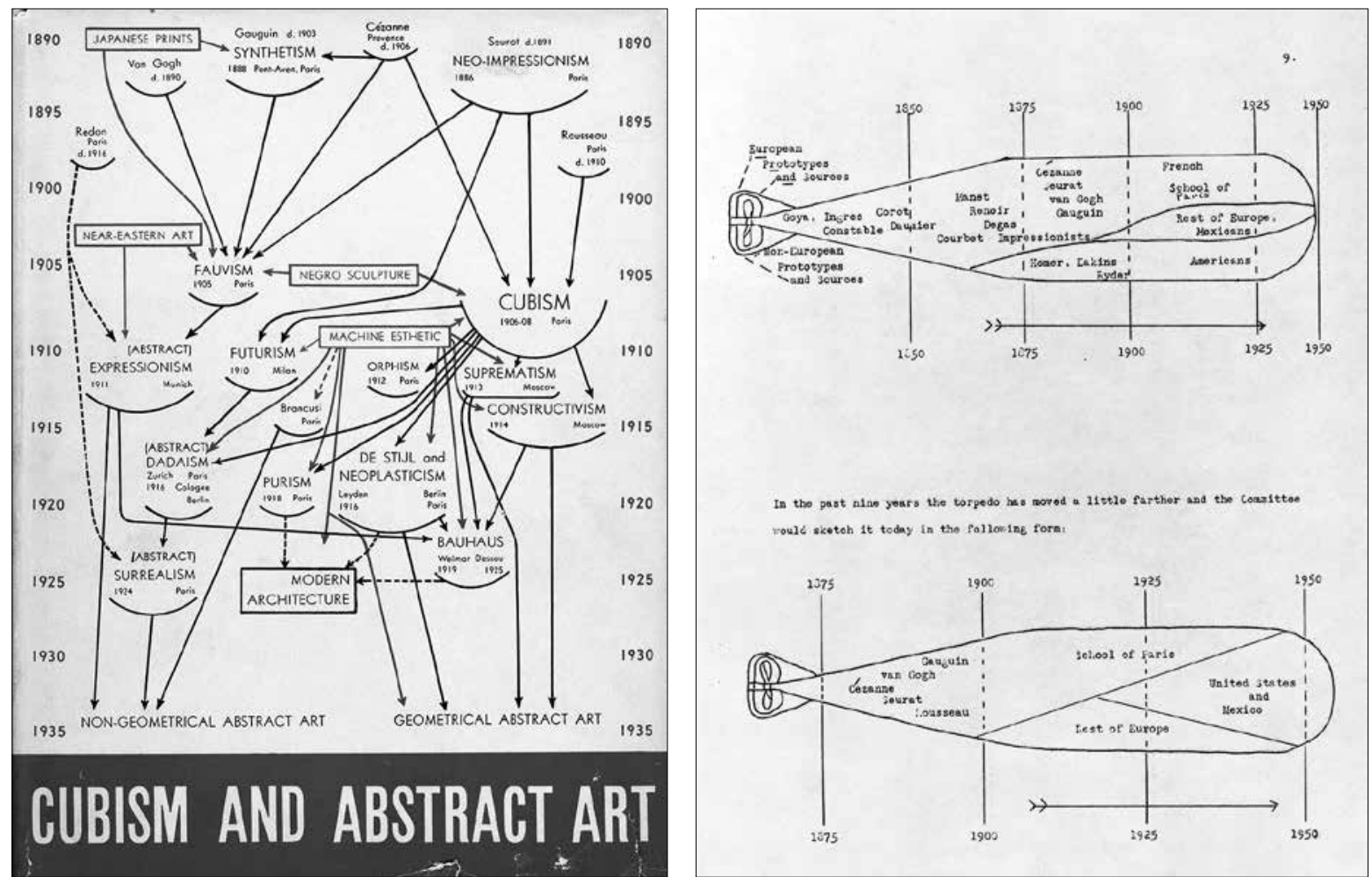
de Francisco de Goya, Jean-Auguste-Dominique Ingres et John Constable jusqu'à l'École de Paris, atteignant son point culminant, comme il aimait le prédire, en 1950, grâce à l'apport des Américains et des Mexicains, le même schéma retravaillé en 1941 montre une histoire différente (fig. 2). Désormais, le mouvement débute avec Paul Cézanne, pousse jusqu'à 1950, et seuls les États-Unis et le Mexique apparaissent comme les détonateurs d'une ère nouvelle. C'était un concept prophétique et programmatique mais, avec notre point de vue actuel, on a l'impression que ces torpilles lancées par le Musée d'art moderne, avec leurs lourdes munitions frontales, après un certain succès initial, se sont finalement écrasées et ont désintégré toute cette organisation qui apparaissait cohérente et irréfutable. De nos jours, tous les morceaux de ce projet moderniste, si bien configuré, sont éparpillés dans le paysage culturel, tandis que le monde occidental repense non seulement la manière de présenter des collections mais aussi, de façon plus large, la raison d'être même des musées d'art moderne. Bien sûr, le monde a changé depuis la chute du mur de Berlin, comme nous l'ont dit les économistes et les politiciens. Le monde est devenu ouvert et global. Les anciennes institutions et autorités qui avaient tiré les ficelles depuis la fin de la Seconde Guerre mondiale sont désormais sérieusement remises en question dans cet âge " postmoderne ». La nouvelle économie globale a en effet transformé les relations internationales tandis que les musées modernes semblent s'être endormis au volant, incapables de réagir à des intérêts si étrangers à leur propre conception historique. De nombreuses voix, ignorées auparavant, commencent à se faire entendre, réclamant d'être pleinement représentées dans les institutions culturelles, créant une large ouverture dans un champ traditionnellement contrôlé par quelques groupes et centres de pouvoir symboliques ${ }^{5}$.

Confrontés à cette nouvelle situation, les musées semblent avoir choisi la voie de sortie la plus facile. S'inscrivant dans la tradition télévisuelle, comme nous l'avons fait remarquer précédemment, les musées pensent désormais que le public (quel que soit le sens que I'on donne à ce terme) veut voir des spectacles de personnalités célèbres : les impressionnistes, Pablo Picasso, Salvador Dalí ou Henri de ToulouseLautrec. Souvent, ces spectacles sont créés par un grand musée appartenant à un centre dominant et sont ensuite loués contre une redevance à d'autres musées plus petits. Nous voilà revenus à l'époque du cirque Barnum qui circulait à travers le pays, s'arrêtant ici et là, éblouissant des gens à la recherche de plaisir avec des animaux étranges et des clowns avant de passer à la prochaine étape. Il est amusant de noter, comme l'a fait Jean Clair, que le musée et le cirque ont été créés à peu près à la même époque, entre 1850 et $1870^{6}$. Ces spectacles itinérants, qui sont désormais la norme, sont trop souvent organisés, sans beaucoup de réflexion mais avec quantité de fanfares et sont expédiés comme des paquets cadeaux. Nous voyons de plus en plus ce genre de productions symbolisant le mépris pour le public.

Le musée devrait être un lieu démocratique, où des débats et des interactions effectives avec les expositions auraient lieu afin d'apporter une meilleure connaissance et une meilleure compréhension de notre propre histoire. Les collections ne devraient pas y être présentées seulement pour montrer à quel point le musée a réussi, grâce à des politiques efficaces et à de l'argent, à s'offrir des grands noms, mais surtout pour expliquer pourquoi ces tableaux qui nous tiennent à cœur ont été importants, le contexte dans lequel ils ont été produits et les idées qu'ils exprimaient dans un environnement donné. De nos jours, les peintures sont présentées comme des collections de timbres : toutes clouées contre un mur, face aux regards vides des visiteurs. Le public, conditionné par les techniques muséales d'accrochage, ne s'excite que lorsqu'il lit les signatures de noms célèbres. Comme le dit Jean Clair : "On se réjouit sans savoir pourquoi, sans savoir ce qu'est la chose pour laquelle nous nous réjouissons. 
Éprouver du plaisir devant une œuvre sans comprendre sa signification, $\mathrm{c}^{\prime}$ est comme jeter un œil à un texte écrit dans une langue étrangère : une série de caractères imprimés, auxquels nous ne comprenons rien ${ }^{7}$.

Si au $\mathrm{XX}^{\mathrm{e}}$ siècle les musées étaient considérés comme élitistes et $d$ 'une certaine manière ennuyeux, ils sont devenus aujourd'hui indispensables pour la visibilité et le marketing de $n^{\prime}$ importe quelle ville ou région ambitieuse. Le terme " musée $\mathrm{d}^{\prime}$ art moderne » donne à n'importe quel assemblage d'objets un vernis culturel brillant, attirant pour une nouvelle population, nombreuse, de touristes versatiles, avides de mimer le « Grand Tour» de l'éducation aristocratique, mais en un laps de temps plus court. D'ailleurs, la

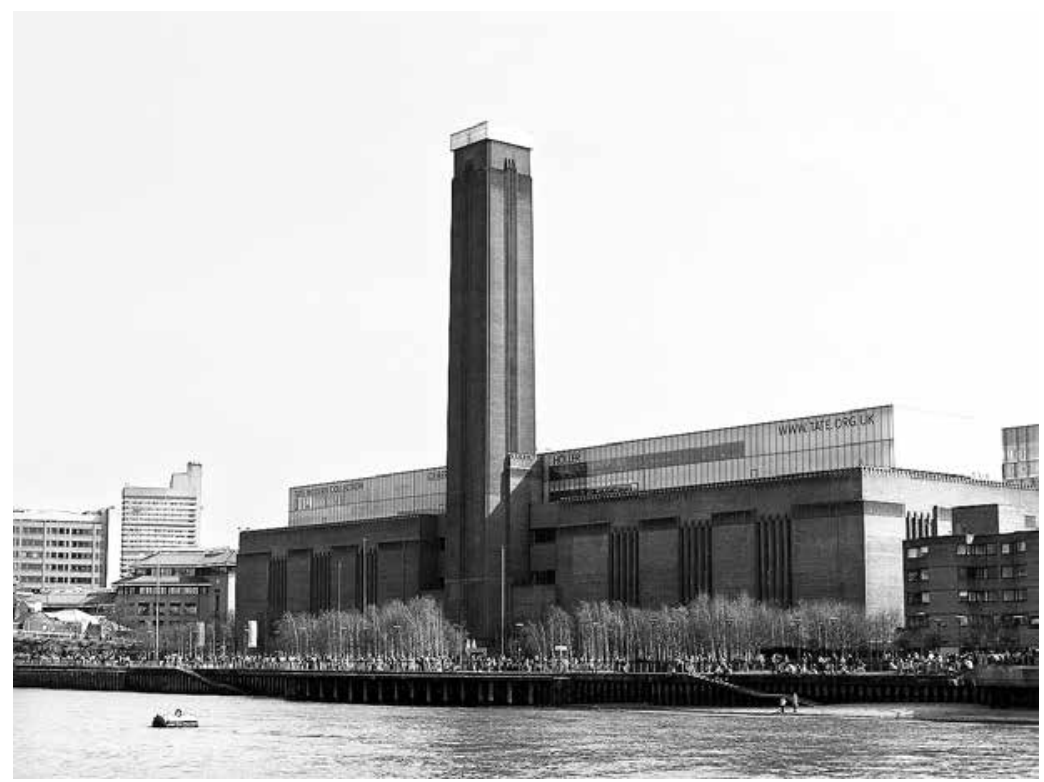
vitesse est essentielle pour ces nouveaux touristes, et la fréquentation des musées pendant ce tour, quels qu'ils soient, l'est tout autant. Les musées, comme un symbole de culture, sont partout; ils se faufilent même dans les anciennes usines et les mines abandonnées. Des bâtiments industriels décrépits et des églises désertées sont transformés en espaces d'art contemporain. On a l'impression que chaque ancien site industriel en friche a été rééquipé et métamorphosé en espèce de musée, les sites les plus ingrats devenant des "lieux de mémoire » consacrés (fig. 3). Des villages ont échangé leur vie quotidienne traditionnelle contre la machine à sous du tourisme saisonnier, annonçant la muséification du monde. Dernièrement, dans le sud de la France par exemple, on pouvait visiter une quantité incroyable de musées divers. Il y en a partout, certains à propos d'art, d'autres d'histoire ou d'artefacts (musées des fers à cheval, des abeilles, du vin, du miel, des fleurs, des senteurs et parfums - il n'est pas nécessaire d'avoir réellement quelque chose à montrer -, musée de la poste, des casseroles, des costumes, des tire-bouchons... et même un musée des crocodiles). À Vancouver, nous pouvons aller sous l'eau pour voir un musée des espèces de poissons en voie de disparition, car le nombre de poissons décroît de manière inversement proportionnelle à la montée du tourisme.

La compétition pour attirer le public - entendu comme la quantité de visiteur-, entre les programmes culturels des villes et les musées traditionnels, est rude, et les musées doivent se faire repérer dans cet environnement concurrentiel. Le musée Guggenheim et ses différentes extensions ont compris quelque chose que les autres musées n'ont pas encore saisi : il faut aller à l'étranger, multiplier ses franchises et s'installer là où se trouve le public (les touristes), plutôt que d'attendre que les touristes viennent à vous, comme l'a fait le MoMA à New York en se reposant sur sa collection réputée et sur la présence d'un nouvel hôtel avoisinant son bâtiment rénové. De nos jours, l'effronterie n'est pas simplement acceptée, elle est en réalité nécessaire. Le Guggenheim a compris que cela crée des dividendes astronomiques de créer des franchises dans d'autres pays; de montrer non seulement, comme toujours, que son propre pays est universel et hégémonique, mais aussi que son implantation à l'étranger renforce l'économie de pays moins riches grâce au tourisme culturel.

3. Tate Modern, ancienne centrale électrique dessinée par Giles Gilbert Scott, reconvertie par le cabinet Herzog \& de Meuron. 


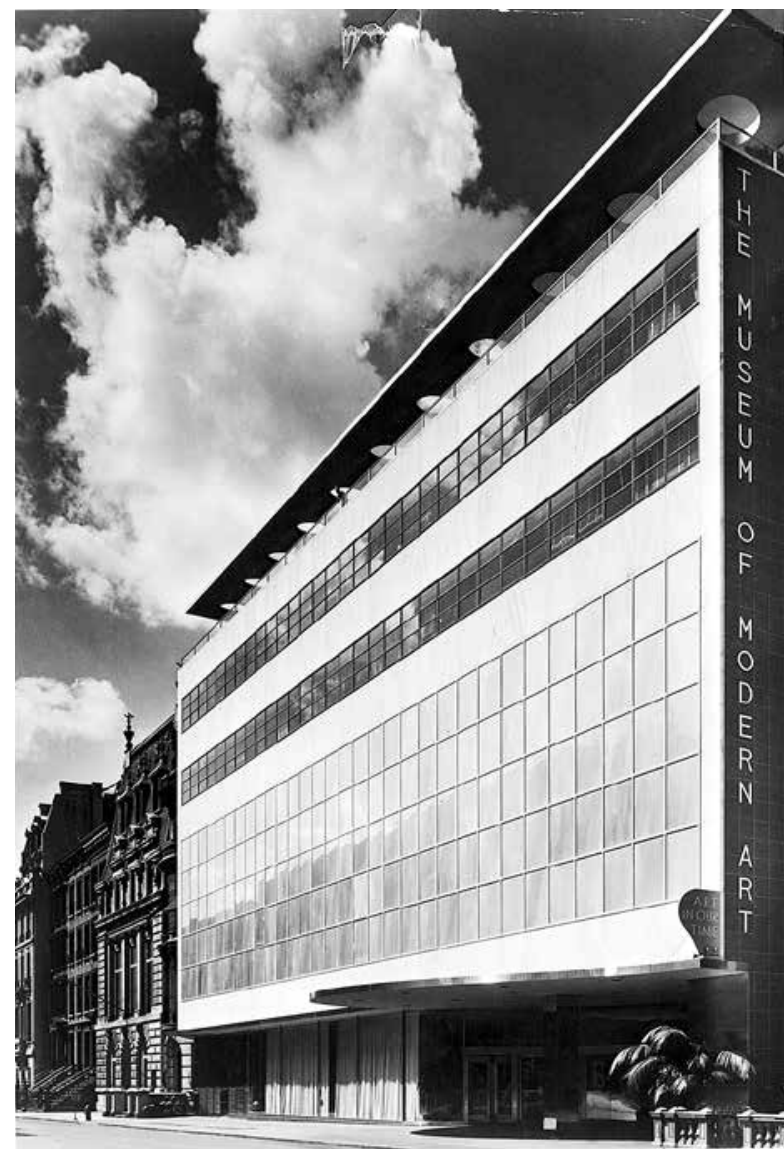

4. Philip L. Goodwin, Edward Durrell Stone, façade du Museum of Modern Art dans sa première forme architecturale, 1939 New York, The Museum of Modern Art Archives, NY. PA58.
$C^{\prime}$ est la manière contemporaine d'utiliser la culture comme un complément de la politique internationale. La politique " du gros bâton » des années passées est désormais remplacée par des signatures d'artistes réputés, y compris celle de Jeff Koons, dont l'ennuyeuse sculpture Puppy, savamment écologique et ironique, monte la garde devant le musée à Bilbao...

Le touriste est désormais la cible principale des musées du XXI e siècle. Mais c'est une cible mouvante. En effet, le touriste moderne est une personne qui voyage rapidement, qui n'a pas de temps mais veut néanmoins faire des expériences "signifiantes » dans un pays étranger. Les cultures locales sont un moyen de rompre avec le quotidien qui devient de plus en plus aliénant et ritualisé. Comme le philosophe français Yves Michaud le fait remarquer : "Les touristes recherchent la liberté, mais c'est une liberté négative qui leur permet de se débarrasser de la routine quotidienne, des obligations, afin de laisser la place au plaisir et à la curiosité. Mais c'est toujours sur une toile de fond de sécurité, qui fait rechercher aux touristes l'exotisme dans la répétition et le cliché. Les touristes souhaitent vivre des expériences, mais en toute sécurité $»^{8}$. C'est du moins ce que supposent les dirigeants des entreprises culturelles. Comme Néstor García Canclini l'a remarqué : «Tous les touristes sont pressés et sont venus jeter un œil sur un paysage, non pas sur l'histoire et le drame local $»^{9}$. Le drame est justement que toutes ces suppositions sont fausses. Les touristes sont aussi actifs, à la recherche de discours alternatifs sur les autres, sur les différents lieux et les différentes cultures. Le problème est que les institutions touristiques, qui incluent désormais les musées, ne procurent pas au public ce qu'il pourrait apprécier - une expérience intéressante et transformatrice - mais préfère des objets fades, rassurants et superficiels. Nous sommes tout près de la notion de kitsch, attaquée il y a des années par le critique d'art américain Clement Greenberg : un vieux problème redevenu central dans le fonctionnement de notre monde global.

Le poids de l'industrie touristique (quatre cents millions d'euros en France d'après les derniers chiffres de 1995) augmente rapidement dans les sociétés économiquement avancées. Il a été multiplié par quatre entre 1960 et 1980. Les revenus du tourisme en France sont par exemple comparables à ceux de l'industrie agro-alimentaire ou de l'automobile. En réalité, le tourisme avec ses deux cents millions d'euros d'activité et ses deux millions d'emplois directs et indirects, est devenu en France le principal secteur $\mathrm{d}^{\prime}$ activité $^{10}$. C'est dans ce contexte, semblable dans tous les pays riches, que les musées d'art moderne essaient de se repenser pour ce nouvel environnement. Leur première réaction est généralement toujours la même : plutôt que de modifier leur structure intellectuelle, ils ont tendance à rénover leur architecture, à agrandir leur espace, à construire de nouvelles ailes afin d'être plus visible à chaque coin des grandes villes, comme un chat marquant son territoire. Le phénomène n'est pas nouveau, mais il est désormais poussé à l'extrême. Déjà en 1939, le MoMA à New York, pour se différencier du vieux monde traditionnel, accentua la transparence, la clarté et le caractère expérimental de l'architecture de son nouveau musée 
pour symboliser ce qu'il représentait : un monde moderne (fig. 4). L'art moderne, rejeté par la majorité des Américains, était protégé et symbolisé par une architecture simple et transparente, exaltant l'individu libre et conquérant, qui va résolument de l'avant. L'accélération de la construction de musées modernes à la fin du XXe siècle montre que l'idéologie des institutions est de plus en plus clairement annoncée dans leur structure architecturale ${ }^{11}$.

Le Centre Pompidou, construit en 1977 et réaménagé en 1985, puis à nouveau en 2000, montre, par sa forme qui rappelle celle d'une raffinerie de pétrole, comment les musées ont réagi aux revendications post-soixante-huitardes de démocratisation et d'ouverture à la culture visuelle contemporaine dans un monde postmoderne. En 2000, la Tate Gallery est transformée pour l'ouverture de la Tate Modern, installée dans une ancienne centrale électrique. Le musée Guggenheim à New York, construit en 1959 à la manière d'une église forteresse, s'est métamorphosé en une éblouissante franchise internationale à Bilbao en 2005. Tous ces musées, comprenant que leur public traditionnel d'amateurs était remplacé progressivement par des touristes pressés, réagirent au changement assez superficiellement à travers leur architecture, sans réellement chercher à moderniser, à repenser leur programmation, malgré les efforts de certains d'entre eux (le Centre Pompidou, la Tate). Dans le cas de Bilbao, les objets d'art conservés dans les réserves du musée new-yorkais ont été transférés et exposés dans la cité basque, une mesure astucieuse qui a transformé des ressources dormantes en une source de profits. À Bilbao, le pouvoir des entreprises américaines est étalé de façon très sophistiquée, non sans rappeler l'ostentation de la tradition du potlatch. Tout est puissamment réuni ici dans la dépense occasionnée par la construction d'un monument somptueux et spectaculaire, coûtant (coût partagé avec le pouvoir local) des sommes très importantes. Mais ce gaspillage traditionnel - la " part maudite » décrite par Georges Bataille - devrait plutôt être appelé la "pars benedicta » (la part bénie) car cette dissipation apparente génère un puissant ensemble de nouveaux circuits économiques (symboliques et réels), mais aussi de la dépendance.

La situation est compliquée par le fait qu'il y a une certaine fierté populaire à être dépositaire d'une culture internationale sexy, et un certain plaisir à être le témoin $d^{\prime}$ un commerce touristique énergique, qui redonne vie à un port industriel en plein déclin situé sur le chemin de pèlerinage qui conduit au site majeur de Saint-Jacquesde-Compostelle. Les deux aspects ne se contredisent pas, en réalité ils se complètent, formant une succession de lieux d'agrément où des reliques de sortes différentes, provenant d'époques diverses, sont offertes à un public impressionné et révérant. Ces deux lieux de dévotion à l'histoire sont tous deux, de façon assez intéressante, hébergés dans des complexes architecturaux dont le but principal est d'éblouir le visiteur. La seule différence majeure est que le musée semble interdire l'activation de la mémoire locale, car l'art et la culture rassemblés sont importés d'un pays étranger. En fait, cette implantation particulière a déclenché un des premiers conflits, ou au moins divergences, entre le global et le local. Néanmoins, avec l'art moderne, les musées d'aujourd'hui sauvent - ou plutôt remplacent - d'anciens espaces industriels en voie $\mathrm{d}^{\prime}$ effondrement par des équipements culturels. Ce processus et ces imbrications du capital global dans le local étaient perçus comme la nouvelle stratégie post-guerre froide de culture de masse globale, d'après la définition de Stuart Hall : " une forme de capital qui reconnaît, pour utiliser une métaphore, qu'il ne peut diriger qu'à travers des capitaux locaux, aux côtés et en partenariat avec les élites politiques et économiques locales $»^{12}$. Mais ce projet, apparemment optimiste, donne rarement complète satisfaction car, comme l'explique John Frow dans son livre Time and Commodity Culture : "Promettant une explosion de modernité, il entraîne aussi un sous- 
développement structurel, à la fois parce qu'il est contrôlé par le capital international et parce que c'est précisément le manque de développement qui rend un lieu attractif pour les touristes ${ }^{13}$. C'est ce qui s'est passé à Bilbao : un grand afflux de touristes mais aussi un désert culturel en dehors de ce centre.

Les nouveaux musées, comme la Tate ou le Guggenheim, symbolisent, du fait de leur taille et de leurs efforts expansionnistes globaux - la Tate dans plusieurs villes $\mathrm{d}^{\prime}$ Angleterre et le Guggenheim presque partout dans le monde (Saint-Pétersbourg, Berlin, Las Vegas, Venise, Rio de Janeiro) -, la culture de la libre entreprise qui est devenue prééminente durant les années 1990. L'artiste Andrea Fraser, commentant avec perspicacité cette transformation, fit la remarque suivante : «Ils ont besoin de grandes expositions et d'œuvres de premier plan pour attirer un large public afin de récolter beaucoup d'argent qui servira à construire de grands espaces pour organiser de grandes expositions avec de grandes œuvres d'art qui attireront un immense public $»^{14}$.

Si vous pensez que je suis trop cynique au sujet de l'avenir des grands musées, laissez-moi vous lire l'invitation que j'ai reçue du Musée des beaux-arts de Vancouver. Le but de cette invitation, comme il est fréquent dans l'univers des musées, est d'attirer les gens à une vente aux enchères d'œuvres d'art appelée "Flash Forward ", dont les bénéfices financeront la construction d'une structure nouvelle, et bien sûr améliorée, pour... en fait nous ne savons pas trop pour quoi. Un nouveau musée clinquant doit être créé pour faire de Vancouver une ville de classe mondiale et un aimant pour les touristes et ses habitants. Quoi qu'il en soit, ce que l'on lit dans l'invitation est une description du type d'activité qui attirera ceux qui ont le niveau de fortune requis : « Le musée est désormais à l'orée de la phase la plus excitante de son histoire. Venez la célébrer avec une vente aux enchères en direct et par écrit, présentant les œuvres des meilleurs artistes canadiens. Suivie d'un cocktail au champagne, d'un diner décadent et de la performance d'un des meilleurs entertainer du moment $»^{15}$. Des célébrités, des paillettes, des bulles, de l'excès et plus encore : l'invitation ressemble à une mise à l'épreuve naïve du nouvel âge des musées. Je bois à sa santé et espère que vous en faites autant.

$\mathrm{Si}$, après la Seconde Guerre mondiale, les États-Unis utilisèrent les films hollywoodiens pour impressionner les populations européennes affamées, avec la mise en scène de I' «American way of life », l'expérience post-guerre froide est celle d'une déterritorialisation extrême des échanges comme des institutions culturelles. Si, dans les années 1950, le salut est venu de la consommation de masse, au XXI ${ }^{\mathrm{e}}$ siècle, il viendra, semblerait-il, du commerce culturel et du tourisme de masse. Comme il est indiqué sur le site internet (intéressant quoique plutôt froid) du critique d'art international et commissaire à la mode Germano Celant qui est - d'une façon caractéristique - à la fois directeur et créateur de la fondazione Prada, la mode et l'art se sont amalgamés : "En fin de compte, tout se marie bien : I'art, la mode, I'architecture, le design, même le shopping. En réalité, tout est du théâtre : un spectacle moderne pour un monde moderne ${ }^{16}$. Cette attitude - un mélange de cynisme et de fatalisme semble assez dangereuse parce qu'elle annihile ce qui était supposé être le rôle du musée moderne depuis sa création : un espace d'éducation et de discussion autour des problèmes posés par l'histoire, par la mémoire ; un espace démocratique pour les arguments et les débats (même si ce but n'a pas toujours été atteint). Avec l'exemple du Guggenheim à Bilbao, tout est réductible à la surface scintillante d'un objet de consommation. On regarde l'architecture, c'est drôle, high-tech, souvent décrit avec humour comme la «tour de Babel », comme une " explosion figée », ou, mieux encore, comme « un Lourdes pour une culture désormais handicapée ». Effectivement, un miracle est peut-être nécessaire pour transformer notre relation avec les musées, mais la bataille sera longue et difficile. 
J'ai déjà été confronté à ce problème à la fin des années 1980 lorsque je discutais de stratégies muséales avec Marcia Tucker, directrice du New Museum of Contemporary Art à New York. Nos positions respectives divergèrent au bout de quelques minutes de discussion. Mon argument était qu'un tel musée devrait combattre la passivité des structures traditionnelles en créant des débats au milieu des expositions. Mais la directrice prônait plutôt un élargissement du canon des œuvres que sa critique. Le musée, $d^{\prime}$ après la directrice, ne pourrait pas faire ce que je proposais afin d'en tester l'ouverture : organiser, par exemple, une exposition d'œuvres d'art que je n'apprécierais pas, tout en expliquant les raisons et en les comparant avec d'autres options. Cette impossibilité a clairement montré que les musées, même les plus anticonformistes, sont seulement des espaces de positivité, d'où critiques et négativité sont bannies, du moins quand cela s'adresse à leurs propres partisans. Même le New Museum, avec toute l'image progressiste qu'il souhaitait projeter, n'avait pas d'autre choix que de faire entendre une voix positive, coincé comme il était entre les égos des artistes, les forces du marché et les traditions historiques ${ }^{17}$. Le New Museum s'est comporté en fait comme les anciens musées, passionnés par les

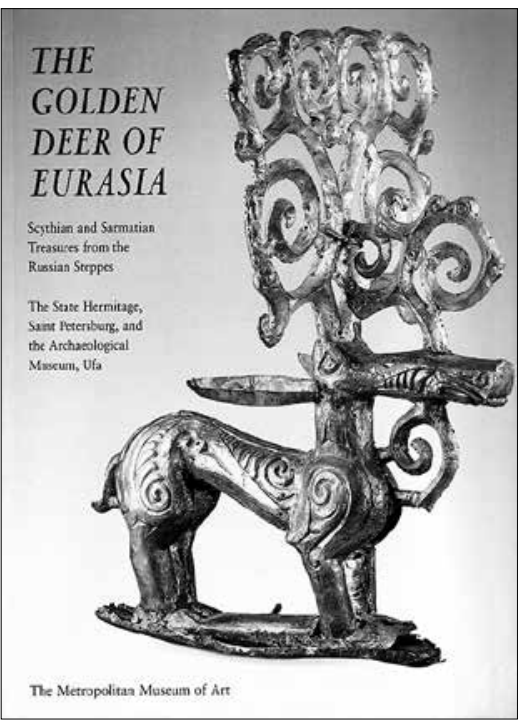
célébrations, I'homogénéité et la positivité, l'opposé en réalité de la vraie vie. Ce n'est donc pas surprenant que le musée soit devenu un des principaux acteurs de l'industrie du divertissement au XXI ${ }^{\mathrm{e}}$ siècle. Comme nous le savons, durant ces dernières années, les expositions internationales, suivant l'exemple donné entre autres par Thomas Hoving au Metropolitan Museum à New York dans les années 1970, sont devenues d'énormes spectacles médiatiques, dans l'optique d'attirer des foules de plus en plus nombreuses. Ces expositions étaient destinées à présenter des trésors universels - en particulier s'ils étaient en or. Rappelons "Treasures of Sacred Maya Kings », "The Golden Deer of Eurasia: Scythian and Sarmatian Treasures from the Steppes ", «Treasures of Tutankhamun », "Princely Splendor: The Dresden Court, 1580-1620 » (fig. 5). Les tableaux en faisaient partie une fois qu'ils avaient été transmués en or à travers leur passage dans les maisons internationales de ventes aux enchères (Van Gogh, Cézanne, les impressionnistes, Pollock, etc.) Ensuite, ayant déjà fait le tour des génies mais toujours tendus vers la compétition et la production de spectacles attractifs, les musées exploitaient les productions artistiques comme si c'était du vin, en offrant différents millésimes : la première période de Cézanne ou de Picasso, leur étape à mi-parcours et leur crépuscule. Parfois c'est le lieu de production qui est exploité, comme Van Gogh à Auvers-sur-Oise ou à Arles. Ces présentations souvent splendides et gigantesques, pleines d'œuvres merveilleuses entassées comme dans une caverne d'Ali Baba, destinées aux amateurs d'art, ne suscitent pas la réflexion du spectateur ou de la spectatrice car il ou elle est rapidement mis à l'écart de toute compréhension du fait du flot de passants, impatients de se rendre à la boutique de cadeaux pour acheter des cartes postales de ce qu'ils n'ont guère eu le temps de regarder... Paradoxalement, ces expositions populaires, produites comme des shows télévisés et conduisant les spectateurs à un état d'hébétude, s'adressent en réalité uniquement aux spécialistes qui ont le bagage suffisant, le savoir qui leur permet de comprendre certaines associations sous-jacentes. Le reste du public réagit souvent comme s'il était hypnotisé par le regard fixe du cobra, endormi et ensorcelé dans un abandon passif.

Si les musées ne sont pas prudents, s'ils veulent participer à de simples jeux de logos qui ne se préoccupent que des apparences, à la recherche de toujours davantage de visiteurs, ils risquent - et peut-être est-ce déjà trop tard - de remplacer le petit espace intellectuel qui reste encore dans les institutions culturelles par un espace qui ressemblera de plus en plus à la télévision américaine, entièrement lisse et sans rides
5. «The Golden Deer of Eurasia: Scythian and Sarmatian Treasures from the Steppes » (New York, Metropolitan Museum of Art, 2000). 
(57 Channels (And Nothin' On) [57 chaînes et rien dedans] comme le chantait Bruce Springsteen en 1992). Si, plus tôt dans le XXe siècle, les musées d'art moderne étaient vus comme des lieux pédagogiques, même si cette éducation était destinée à une minorité privilégiée, il semblerait qu'ils soient désormais des centres de divertissement, d'où sont bannis à nouveau les débats : cette fois-ci non pas à cause de l'intimidant silence religieux des premiers temps mais à cause de l'intense cacophonie, cette activité tourbillonnante joyeuse et vide qui interdit toute attitude profonde et réflexive. Avec ces nouvelles structures, les musées risquent de produire une génération incapable de débattre, ou de comprendre les enjeux politiques et culturels, incapable, finalement, de participer au débat démocratique. Ce n'est pas Internet tout seul qui proposera une alternative critique efficace.

Être sérieux au sujet de l'art signifie essayer de montrer combien il est difficile de produire des déclarations artistiques, de réagir aux questions importantes sur la représentation ou sur la compréhension personnelle des questions historiques à une époque précise. Les artistes et les intellectuels ne produisent pas des images et des textes sans raison ou simplement pour s'amuser. Isoler l'art du questionnement, de son engagement dans les débats - en dépit de ce que les esthètes, qui veulent juste regarder des images, en disent -, c'est dénigrer l'art et diminuer son importance. Poussons alors les musées à avoir une relation adulte avec leurs publics ; demandonsleur de réfléchir au sujet de ce nouveau public et de ses besoins importants. Ce que les musées ont perdu, c'est ce qui les distinguait du divertissement : un engagement sérieux aux côtés de l'histoire et de la mémoire. Certes, I'histoire est aussi une sorte de construction, mais, si le regard historique ne représente pas non plus la vérité, il est moins factice que le divertissement parce qu'il met en avant la pensée, la réflexion et une certaine forme d'engagement, de conscience de soi-même. Est-ce que le temps n'est pas venu d'oublier le vieux syndrome du Kunstkammer, quand les objets rares étaient tous regroupés, indifféremment, et utilisés comme des trophées, des symboles de pouvoir? Le temps n'est-il pas venu de créer un espace où le public pourrait instaurer un dialogue, avec une interprétation affichée de l'histoire, sur les murs du musée, une thèse en d'autres termes ? Le temps n'est-il pas venu de donner, dans l'élaboration d'une exposition (exhibition en anglais, d'où exhibitionnisme, frime), suffisamment de matière pour que les visiteurs puissent saisir ce qui était en jeu dans le passé et comment certaines solutions étaient recherchées, certains choix faits ? Le temps n'est-il pas venu pour les musées de devenir des centres de recherche, plutôt que des halls d'exposition de produits?

Il est d'ailleurs intéressant de remarquer que pendant les trente dernières années, nous avons vu une certaine transformation du chercheur dans le domaine culturel, qui ne veut plus créer un empire (fixant les significations pour lui-même ou elle-même) comme Michel De Certeau l'a décrit, mais qui veut donner une analyse critique des lois qui organisent les faits constatés. Les musées ont besoin de renouer avec la recherche, d'être partie prenante du dialogue culturel et politique. La recherche devrait être intégrée avec beaucoup plus de détermination dans les expositions des musées, et devrait permettre de déstabiliser les certitudes en comprenant la façon dont d'autres, à différentes époques, ont parlé, ont mis en place des stratégies, ont été lus et ont compris. Il faut constamment relire les constructions passées à travers la compréhension ou les besoins actuels. La recherche fait partie de la bataille pour la signification, participe de la lutte pour articuler un type d'histoire, en opposition au statu quo, qui présente des visions du monde différentes, complexes et contradictoires. La recherche introduite dans les expositions est, en réalité, faite pour aujourd'hui, elle contribue activement à notre compréhension des discours culturels. C'est pourquoi Paul Virilio, dans l'un de ses derniers entretiens, 
explique que le rôle de l'intellectuel et du chercheur est de développer des discours antagonistes contrairement à la tendance actuelle à tout pacifier, à tout traiter au prisme de la démagogie.

Il est temps de relire les descriptions canoniques, selon des points de vue enrichis par la juxtaposition de différents documents, ou en s'appuyant sur un autre regard, de traverse, afin de percevoir les possibilités et les lectures différentes. Cette approche serait une histoire libérée de la domination des institutions qui essaient fébrilement d'utiliser leurs propriétés - les collections - afin d'écrire, comme le prince avait l'habitude de le faire autrefois, leur propre histoire de la manière appropriée : leur manière, la seule manière, comme le MoMA l'a fait récemment, engageant les services d'une plume apparemment obéissante pour réécrire une histoire embellie de la période de l'après-guerre ${ }^{18}$.

\section{Concernant la Nouvelle Histoire}

Ces dernières années, les historiens se sont démenés pour négocier de nombreux tournants, tellement nombreux (le tournant sémiotique, le tournant culturel, le tournant linguistique) que la profession a commencé à montrer des signes de vertige ${ }^{19}$. De même, par ricochet, I'histoire de l'art. C'est le moment idéal pour les historiens de comprendre que, depuis l'origine, les œuvres d'art, les productions culturelles et les images n'ont pas été données, mais construites avec de l'incertitude et des contradictions. Ces constructions complexes sont devenues, pour les historiens de l'art, le chantier privilégié où les forces sociales, à travers la subjectivité de l'artiste, s'affrontent ou dialoguent. C'est de là également que l'historien tirera désormais les multiples explications suscitées par l'œuvre d'art, lorsqu'elle est confrontée aux différentes lectures proposées par des publics variés, défendant souvent des intérêts contradictoires. Ce qui donne de la force aux recherches contemporaines en histoire de l'art ces derniers temps, est le fait qu'en fin de compte, après tant d'années d'isolement, le champ s'est ouvert aux études transversales et, en dehors du champ, à des expériences qui conduisent à déstabiliser d'anciennes certitudes, ainsi que des traditions politiques et esthétiques.

C'est ce que j'ai essayé de faire avec l'aide de Manuel Borja-Villel - la traduction de la recherche dans un dialogue avec le public - dans la production d'une exposition intitulée Be-bomb: The Transatlantic War of Images and All That Jazz, 1946-1956 pour le Museu d'Art Contemporani de Barcelona en 2007 (fig. 6) ${ }^{20}$. Cette exposition décrit les débats et les questions autour des relations culturelles et politiques, assez turbulentes à cette époque, entre Paris et New York, de 1944 à 1956. Depuis le début du projet, mon idée était d'essayer de comprendre et de présenter au public les tentatives très différentes visant à reconstruire un pouvoir culturel occidental hégémonique, sur les ruines de la Seconde Guerre mondiale. Après des recherches dans le domaine littéraire, et l'exploration de kilomètres d'archives sur les deux continents, il me parut évident que cette histoire de reconstruction reposait sur des subtilités et nécessitait un recours à plusieurs langues, afin d'envisager les différents angles, en mettant de nombreux nouveaux outils à la disposition du chercheur. Il était clair que les arts visuels étaient très importants pour l'identité nationale, mais c'était aussi le cas du jazz. Ce dernier en fait devint une façon de critiquer, à travers son élévation au statut de " classique ", la société américaine. De la même manière, la construction et la signification d'un type de comportement social et de style de vie alternatifs développés dans le vieux Quartier latin de Paris, furent cruciaux pour la définition d'une nouvelle culture française, de même que la diatribe
6. Vue de l'exposition Be-bomb: The Transatlantic War of Images and All That Jazz, 1946-1956 (Barcelone, Museu d'Art Contemporani de Barcelona, 2007).

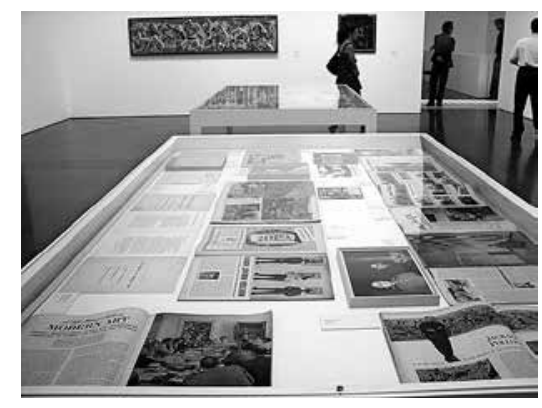


de 1947 autour du style New Look de Christian Dior et le violent débat de 1948 autour du mot «moderne » aux États-Unis. On n'oubliera pas non plus l'importance de I'explosion atomique sur I'atoll de Bikini, tandis que la guerre froide se profilait à l'horizon politique. La recherche fut multiforme, multidirectionnelle et amusante à mener, avec souvent des découvertes surprenantes, mais elle présentait pas mal de difficultés, au final, au moment de mettre en scène les résultats sur les murs et dans les espaces du musée. C'était un défi pour toutes sortes de raisons qui n'avaient rien à voir avec le large éventail de recherches effectuées, ni avec les propres désirs du musée, mais plutôt avec des problèmes de coût, de localisation, d'emprunt d'œuvres et $d^{\prime}$ autres questions administratives.

Néanmoins, décoloniser l'œil occidental était l'un des objectifs principaux de la recherche dans laquelle je me suis lancé pour cette exposition. La recherche, c'est-à-dire la collecte de documents et objets divers et nouveaux en histoire de l'art, fut élaborée dans le but de déployer publiquement les débats différemment construits qui, comme dans ces deux pays, pour des raisons différentes, ont structuré la manière de comprendre la culture moderne d'après-guerre. La recherche insistait sur les modèles comparatifs, car il était important de confronter de manière synchronique, dans les deux pays, les constructions politiques et culturelles internes, ainsi que de comparer deux approches différentes dans les affaires internationales. L'idée sousjacente à l'exposition était de nous laisser - nous les spectateurs intéressés - nous promener dans la culture de l'immédiat après-guerre sans ces œillères formalistes érigées par New York (ou alors Paris), œillères qui, depuis des décennies, ont cantonné le regard des amateurs dans un cadre intellectuel très limité. La chronologie courte et spécifique de l'exposition permit, à travers les éléments dénichés pendant les recherches, de présenter des débats d'opinion divers et animés autour de l'identité nationale, I'histoire et le pouvoir à une période où les échanges étaient certes dangereux, mais encore démocratiques. Cela donna aussi la possibilité d'approfondir sur chaque scène artistique afin d'exhumer une histoire dense et parfois non conventionnelle. L'exposition présentait sur ses murs une discussion autour d'un moment spécifique, synchronique, à travers un éventail de différentes formes d'expression : peinture, mode, musique, film, mis en parallèle avec les événements du moment, afin de montrer comment ces éléments, à une époque donnée et à travers des dialogues spécifiques, contribuèrent à une réorganisation plus vaste et concurrentielle du monde occidental.

Effectivement, le projet d'exposition n'était pas de présenter seulement une succession d'œuvres d'artistes célèbres et triomphants, comme c'est malheureusement trop souvent le cas dans les expositions, mais, à l'inverse, de les immerger au sein de débats essentiels dans lesquels ces œuvres d'art ont été, en réalité, directement ou indirectement impliquées. Les œuvres d'art étaient " mises en situation » comme on dit, brisant simultanément le lieu saint du cube blanc muséal et la camisole de force intellectuelle que sont les traditions formalistes ou de connoisseurship, désormais bien connues. Nous espérions, en introduisant des discours autres que ceux de la peinture et de la sculpture au sein de l'espace du musée, que les œuvres $d$ 'art seraient vues et comprises comme une part vitale du vaste et excitant dialogue autour de l'identité nationale et du positionnement individuel et social pendant ce moment important de réorganisation générale sous la pression de la guerre froide. Dans ce contexte, des activités et des déclarations d'avant-garde furent intentionnellement juxtaposées avec des propos officiels et traditionalistes, afin de montrer à quel point les choix esthétiques étaient controversés et revêtaient de l'importance au moment de la guerre froide, alors que les symboles et la propagande étaient au centre de toutes les attentions. 
De diverses manières, ce type de recherche multidirectionnelle nous permettait d'un côté de redonner vie à des tableaux célèbres, qui, du fait de leur célébrité, existaient comme pétrifiés dans un espace sans signification, vidés de leur sens originel et essentiel, à la faveur d'une signature dotée d'une valeur marchande. De l'autre côté, il était également important de montrer la place historiquement riche de sens d'autres œuvres, qui avaient été oubliées et effacées de l'histoire de l'art canonique et linéaire, sans créer un autre renversement de perspective révisionniste.

La résurgence régulière de l'espace neutre traditionnel du musée dans l'exposition était conçue pour laisser parler ces œuvres mais cette fois pas dans le vide : elles devaient parler grâce à la compréhension de leur discours, construite et accumulée par le visiteur dans les salles précédentes - autrement dit "en connaissance de cause ». Ces salles " de réflexion », comme nous les avions appelées, donnaient la possibilité au visiteur de lire et d'apprécier les messages, les interrogations, traduites en images par des artistes dialoguant avec leur propre époque et I'histoire de leur propre métier, avec toutes les complexités et contradictions que cela implique. Tout cela parce que la visite d'une exposition ne devrait pas être seulement une expérience contemplative mais plutôt l'occasion de s'émerveiller et de déambuler, naviguant dans l'épaisseur du passé jusqu'à permettre une réévaluation des positions des spectateurs qui, avec un peu de chance, seront surpris et enchantés par les nouvelles possibilités de lecture et les découvertes inattendues. C'est ce que j'appelle un environnement démocratique, où le passé est décrit afin de favoriser notre questionnement, $d^{\prime}$ interroger la manière dont il a été traduit, construit et encadré par de nombreuses tendances idéologiques. Le but de l'exercice, l'objectif d'une exposition, devrait être de remettre en question des lectures passées, et non pas d'affirmer des clichés existants. C'est pour cette simple raison que la fameuse torpille d'Alfred Barr devrait être retirée de la circulation, voire explosée, afin de laisser le champ libre pour reconfigurer le puzzle du passé, sans crainte et dans l'allégresse. Les musées ont le pouvoir de faire cela, mais en ont-ils la volonté ? Je n'en suis pas sûr, sachant (et voyant) ce qui est en réserve pour le futur, un futur qui semble être déjà arrivé. Connaissant la tendance touristique de notre culture, allons-nous regarder les œuvres d'art célèbres de la même façon que le pèlerin à Mexico vient voir la révérée Vierge de la Guadalupe ? Imaginez cela au Musée du Louvre devant La Joconde ou à Madrid devant Guernica. À Mexico, les autorités, confrontées au problème fastidieux de la gestion des masses, ce mélange fatal de pèlerins et de touristes, ont décidé d'installer un trottoir roulant pour gérer le flux des visiteurs passant devant l'image de la sainte (fig. 7). Le culte contemporain de la vitesse crée des ravages parmi les pèlerins, qui non seulement ont l'habitude $d^{\prime}$ 'une atmosphère plus contemplative, mais espèrent aussi être en mesure d'apercevoir le fameux regard miraculeux de la sainte. Bienvenue donc dans ce nouveau monde où les logos, la vitesse, la vacuité et la publicité, non seulement dirigent et contrôlent notre regard mais désormais, avec ce sacré exemple, littéralement mènent la danse.

Ce texte a fait l'objet d'une conférence qui a eu lieu le 5 novembre 2014 à I'INHA. Il a été traduit par Isabelle Dubois.

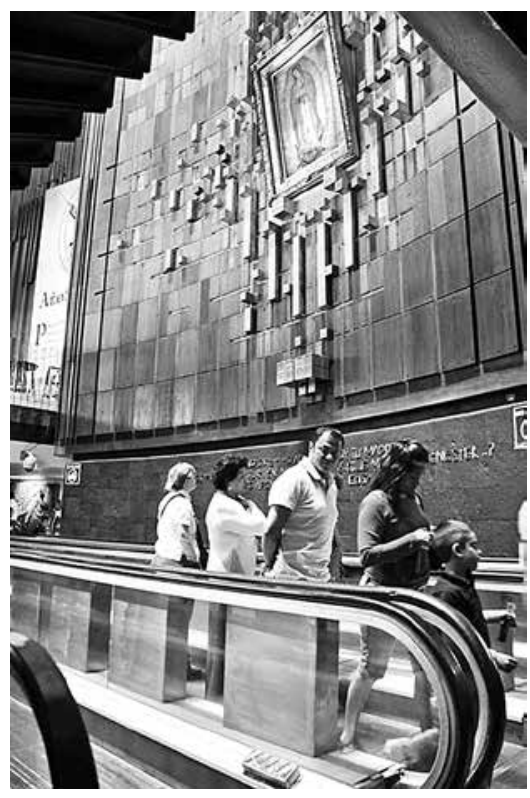


1. Jean Clair, Malaise dans les musées, Paris, 2007, p. 134.

2. Clair, 2007, cité n. 1, p. 37.

3. Cité dans Clair, 2007, cité n. 1, p. 56 (d'après le rapport de Jean-Pierre Jouyet et Maurice Lévy, L'Économie de l'immatériel, Paris, 2006, p. 123).

4. Voir l'excellente étude de Jesús Pedro Lorente, Les Musées d'art moderne et contemporain : une exploration conceptuelle et historique, Paris, 2009 ; Marcelo Expósito, Conversación con Manuel BorjaVillel, Madrid, 2015. Plus tard, j'ai été heureux de voir que le Musée national d'art moderne-Centre Pompidou avait changé la présentation de ses collections à travers un important apport d'œuvres. Le voyage chronologique proposé par des salles de recherche où des documents révèlent la force de la critique d'art (appelée " passeur ") à définir - avec l'aide des artistes - de nouveaux enjeux et directions : un formidable changement de l'art moderne dans les musées qui aidera à comprendre sa complexité et son histoire à plusieurs niveaux.

5. Une avalanche de livres essaye désormais de traiter du rôle des musées de nos jours mais aussi des nouvelles pratiques des conservateurs. Voir Ivan Karp, Stephen D. Lavine éd., Exhibiting Cultures: The Poetics and Politics of Museum Displays, Washington, D.C., 1990 ; Antoni Muntadas, Between the Frames: transcriptions des entretiens, Bordeaux, 1994 ; The end(s) of the Museum=Els límits del museum, Thomas Keenan, Alexander García Düttmann éd., (cat. expo., Barcelone, Fundació Antoni Tàpies, 1994), Barcelone, 1996 ; Laurent Gervereau éd., Musées et politique, (colloque, Québec, 1998), Québec, 1999 ; Melanie Townsend éd., Beyond the Box: Diverging Curatorial Practices, Banff, 2003 ; Maurice Berger éd., Museums of Tomorrow: a Virtual Discussion, (colloque, Santa Fe, 2003), Baltimore/Santa Fe/New York, 2004 ; numéro thématique Du musée au parc d'attractions : ambivalence des formes de l'exposition, sous la direction de Serge Chaumier, de Culture et Musées, 5, 2005 ; Lorente, 2009, cité n. 4.

6. Clair, 2007, cité n. 1, p. 69.

7. Clair, 2007, cité n. 1, p. 95.

8. "Tourists seek freedom, although this is a negative freedom that allows them to rid themselves of everydayness, routine, obligations, so as to make room for pleasure and curiosity. Yet this is always against a backdrop of security, which makes the tourist seek exoticism in repetition and the cliché. The tourist wishes to live the experience with security » (Yves Michaud, "Looking beyond the challenges of rural tourism ", dans New Policies for Cultural Tourism: challenges, ruptures, responses, numéro thématique de Nexus, 35, hiver 2005-2006, p. 4).

9. "All tourists are in a rush and come to get to know an eye-catching landscape - not the history of local drama » (Néstor García Canclini,
"Paranoia vs. a utilitarian vision ", dans Nexus, 2005-2006, cité n. 8, p. 6).

10. Voir Michaud, 2005-2006, cité n. 8, p. 4.

11. Voir Anna Maria Guasch, Joseba Zulaika éd., Learning from the Bilbao Guggenheim, (colloque, Reno, 2004), Reno, 2005.

12. " [...] now a form of capital which recognizes that it can only, to use a metaphor, rule through other local capitals, rule alongside and in partnership with other economic and political elites " (Stuart Hall, " the Local and the Global: Globalization and Ethnicity ", dans Anthony D. King éd., Culture, Globalization, and the World-System: Contemporary Conditions for the Representation of Identity, Minneapolis, 1997, p. 28).

13. "Promising an explosion of modernity, it brings about structural underdevelopment, both because of its control by international capital and 'because it is precisely the lack of development which makes an area attractive as a tourist goal' " (John Frow, Time and Commodity Culture: Essays in Cultural Theory and Postmodernity, Oxford/New York, 1997, p. 101).

14. Andrea Fraser, conférence tenue à l'University of British Columbia à l'occasion de l'exposition qui lui a été consacrée à la Morris and Helen Belkin Art Gallery à Vancouver en février 2004.

15. " the Gallery stands on the edge of the most electrifying phase yet. Come celebrate with a live and silent auction featuring works by many of Canada's finest artists. Party with champagne reception, decadent dinner, dance and performance by one of today's most fêted entertainers ».

16. "But in the end it all fits together: art, fashion, architecture, design - even shopping. It's all theatre, really. A modern spectacle for a modern world " (Michael Specter, "The Designer », dans The New Yorker, 15 mars, 2004, p. 111).

17. Pour une excellente analyse des difficultés rencontrées par un directeur de musée, voir les mémoires de Marcia Tucker, A Short Life of Trouble: Forty Years in the New York Art World, Berkeley/Los Angeles, 2008.

18. Voir en particulier Michael Kimmelman, «Revisiting the Revisionists: The Modern, Its Critics and the Cold War ", dans The Museum of Modern Art at Mid-Century, at Home and Abroad, 4, New York, 1994.

19. Voir le livre pertinent, édité par Gabrielle M. Spiegel, Practicing History: New Directions in Historical Writing After the Linguistic Turn, New York, 2005.

20. Be-bomb: The Transatlantic War of Images and All That Jazz, 1946-1956, Serge Guilbaut, Manuel Borja-Villel éd., (cat. expo., Barcelone, Museu d'Art Contemporani de Barcelona, 2007-2008), Barcelone, 2007. 